\title{
The apoptotic pore on mitochondria: are we breaking through or still stuck?
}

\author{
C Borner ${ }^{*, 1}$ and DW Andrews ${ }^{2}$ \\ Cell Death and Differentiation (2014) 21, 187-191; doi:10.1038/cdd.2013.169
}

The past 25 years of research has brought us close to understanding the apoptotic form of programmed cell death, a major regulatory mechanism that shapes us during embryogenesis and keeps us properly functional and healthy on a daily basis. We know that mistakes in regulating this process contribute to various human diseases ranging from cancer, autoimmunity, immunodeficiency to neurodegenerative diseases, and we now have novel drugs targeting apoptosisregulatory molecules in development or at hand. Some of these compounds have entered clinical trials and might be beneficial in curing diseases such as cancer, particularly in combination with other classical therapeutics.

Apoptosis proceeds through two major signaling pathways, an extrinsic pathway initiated by TNF-like death ligands and an intrinsic pathway involving mitochondrial outer membrane permeabilization (MOMP) triggered by a variety of apoptotic stimuli, such as chemotherapeutic drugs, irradiation, the deprivation of growth factors/cytokines or cell-matrix interactions and others. The molecular mechanisms involved in extrinsic apoptosis signaling induced by TNF $\alpha$ and its related cousin FasL have been largely defined, and basically involve the clustering of their respective receptors and the recruitment and activation of initiator caspases- 8 and 10 via the FADD adapter molecule and the subsequent cleavage and activation of the effector caspases- 3 and 7 by the initiator caspases. It is also well known that in some particular cells, the extrinsic pathway can crosstalk with the intrinsic pathway via cleavage of the $\mathrm{BH} 3$ protein Bid by caspase-8. What might be a deviation of the theme is the signaling pathway induced by the TNF-like ligand TRAIL. In this case, yet unidentified molecules may modulate the caspase-8/caspase- 3 activation axis. Moreover, caspase- 8 has recently become implicated in novel death signaling pathways that emerge from within the cell such as after viral infection, glucose deprivation or the induction of autophagy. Finally, we know now that under caspase-inhibiting conditions, TNF $\alpha$ can also activate alternative death signaling such as necroptosis, which involves RIP-like protein kinases that may crosstalk with mitochondria.

Intrinsic, mitochondria-mediated apoptosis signaling is tightly regulated by members of the Bcl-2 family. A subgroup of the family, the BH3 proteins both 'sense' the apoptotic stimuli by posttranscriptional mechanisms and function as the mediators of transcriptional responses. The full scope of the posttranscriptional mechanisms has not yet been unveiled, but we know that it often exposes and/or structures a particular domain in these proteins, called the BH3 domain, which then is able to activate MOMP via two basic strategies: (i) to bind to the hydrophobic pocket of Bcl-2-like survival factors, such as Bcl-2, Bcl- $x_{\mathrm{L}}, \mathrm{Bcl}-\mathrm{w}, \mathrm{Mcl}-1$ and A1, inhibiting them and thereby releasing the pre-bound third subclass of the family, the Bax and Bak effectors, or (ii) to directly activate Bax and Bak on the mitochondrial membrane. The latter strategy is thought to be limited to three particular $\mathrm{BH} 3$ proteins, called tBid, Bim and Puma, which are either activated transcriptionally, by phosphorylation (see recent publications in Cell Death and Differentiation ${ }^{1,2}$ ) or by proteolytic cleavage. The consequence of Bax/Bak activation is MOMP, which leads to the release of apoptogenic factors from the intermembrane space of mitochondria to either trigger caspase-independent processes or activate caspases-3/-7 via the formation of an Apaf-1/caspase-9-containing apoptosome.

There is general agreement regarding the involvement of $\mathrm{BH} 3$ proteins to directly or indirectly activate Bax/Bak and the participation of $\mathrm{Bcl}-2$-like survival factors in inhibiting or finetuning this process. However, as highlighted in two reviews in the current issue of Cell Death and Differentiation, ${ }^{3,4}$ there is little consent regarding the exact molecular mechanisms by which $\mathrm{BH} 3$ proteins interact with and indeed activate Bax and Bak, and how then Bax and Bak provoke MOMP. As a result cogent arguments can be presented that are almost diametrically opposite regarding the composition and structure of the molecular complex that permeabilizes the outer membrane of mitochondria. Indeed even the stated facts used as starting points in the reviews appear mutually exclusive. Here we highlight some of these controversies as three broad questions. (1) What is the molecular composition of the permeabilizing structure? (2) How is it assembled? And (3) how do our experimental approaches bias our understanding?

\section{What is the molecular composition of the Bax/Bak permeabilizing structure and is it fixed?}

MOMP releases apoptogenic proteins from the intermembrane space of mitochondria into the cytoplasm. However,

\footnotetext{
${ }^{1}$ Institute of Molecular Medicine and Spemann Graduate School of Biology and Medicine (SGBM), Albert Ludwigs University Freiburg, Stefan-Meier-Strasse 17, D-79104 Freiburg, Germany and 'Sunnybrook Research Institute, Department of Biochemistry, University of Toronto, 2075 Bayview Avenue, M7-640, Toronto, ON, Canada, M4N 3M5

${ }^{*}$ Corresponding author: C Borner, Medical Cell Research, Institute of Molecular Medicine, Stefan-Meier-Strasse 17, D-79104 Freiburg, Germany. Tel: +49 761203 9618; Fax: +449 761203 9620; E-mail: christoph.borner@uniklinik-freiburg.de
} 
there is no experimental proof that permeabilization is mediated by either a lipidic (Figures $1 a$ and b; labeled 2) or proteinaceous pore (Figures 1a and b; labeled 1) in the outer mitochondrial membrane. Experiments using nanodics (protein delimited planar lipid bilayers) suggest that when Bax binds to membranes, it unfolds on the surface similar to an umbrella. Such a conformation is not compatible with the formation of a protein-lined pore, leading some groups to search for and find evidence of a lipidic pore. Furthermore, some kinetic measurements suggest that Bax monomers, not oligomers, are rate-limiting in driving membrane perturbation and/or pore formation. But what would then be the role of Bax/Bak dimerization or even multimerization? There is abundant evidence that membrane-bound Bax oligomers 'auto-activate' monomeric soluble Bax. Would there be kinetic or thermodynamic advantages of such a mechanism to merge lipidic pores as opposed to enlarging protein-lined pores? Kinetic arguments provide compelling tests of specific models but are rarely completely unambiguous. Similarly, the widely described observation that mutations in Bax or Bak that block oligomerization also block MOMP does not necessarily mean that permeabilization sites must be composed of oligomers of these proteins. Membrane insertion of Bax could be the rate-limiting step of MOMP resulting in the observed linear kinetics of the process. ${ }^{5}$ On the other hand, oligomers might not be the permeabilizing entity but may bind and inhibit anti-apoptotic proteins.

It appears that MOMP often requires an interaction between the regulatory $\mathrm{BH} 3$ proteins and Bax or Bak to initiate the process. The interaction is generally regarded as transient and is most often described as 'hit and run' to account for $\mathrm{BH} 3$ proteins being absent from oligomers of $\mathrm{Bax}$ and Bak solubilized from mitochondria. However, the molecular basis for what is meant by the phrase 'hitand-run' is not well defined. It could indicate only that the interaction is reversible and has a relatively low affinity in detergent-solubilized membranes. Alternatively, either protein could undergo a conformational change in response to the interaction that dramatically reduces the binding affinity such that re-binding even in membranes is highly unlikely. Furthermore, it is unclear if activation of Bax universally requires interaction with a $\mathrm{BH} 3$ protein. It may also be achieved by physiochemical changes such as $\mathrm{pH}$ or redox potential.
If the interactions between $\mathrm{Bcl}-2$ family proteins are governed by standard equilibria, as are the vast majority of protein-protein interactions, then the composition of the membrane-permeabilizing complex may not be fixed or static. For example, tBid binding to Bax in membranes has a reported apparent $K_{D}$ of $25 \mathrm{nM}$ while binding can barely be detected for the proteins in solution. At present, there is no consistent experimental evidence for the stoichiometry of any of the pore components. Estimates of the number of Bax proteins in membrane-permeabilizing complexes range from one (as discussed by Volkmann et al. ${ }^{3}$ ) to hundreds. ${ }^{6}$ And that is just for Bax. As Bcl- $x_{\mathrm{L}}$ appears to function more or less like a dominant negative Bax, it is possible that in membranes, aborted Bax oligomeric complexes can contain several different $\mathrm{Bcl}-2$ family proteins.

\section{How is a Bax/Bak permeabilization site assembled?}

Given the uncertainties described above, it is not surprising that the mechanism of activation of Bax and Bak by $\mathrm{BH} 3$ proteins is also uncertain. The Walensky group has generated convincing data suggesting that at least Bax has two activation surfaces. ${ }^{7}$ Dual activation surfaces, whether hierarchical or not, provide an attractive explanation for the growth of oligomers. A single interaction site would not readily support the generation of oligomers larger than dimers.

Irrespective of whether the active forms of Bax or Bak are of monomeric or oligomeric nature, the question remains, how exactly each of the known $\mathrm{BH} 3$ proteins brings about their activation state. For years, the apoptosis community tried to prove two highly disputed activation model systems. The 'direct activation' model claims that certain BH3 proteins, the so-called 'direct activators' tBid, Bim and Puma, are not only able to bind the hydrophobic groove of Bcl-2-like survival factors to be neutralized, but can also activate Bax/Bak by direct physical contact (Figure 1a, right half). The best experimental and structural evidence for such a mechanism comes from studies with tBid and Bim, less so from Puma. The 'indirect activation or derepression' model suggests that Bax and Bak are both bound to Bcl-2-like survival factors in healthy cells (Figure 1a, left half). $\mathrm{BH} 3$ proteins, after their activation upon apoptotic stimuli and insertion into the MOM, would release Bax/Bak from inhibition by competing with the same

Figure 1 Models and MODES of Bax/Bak activation and presumed formation of the apoptotic pore. (a) The previously proposed 'derepression' model suggests that Bak is kept in check by Bcl-2-like survival factors on the MOM in healthy cells. Upon apoptosis stimulation, BH3 proteins (all) become activated and displace Bak from its inhibition, activating it autonomously. How Bax is activated in this model remains enigmatic as Bax/Bcl-2-like complexes are rarely seen in healthy cells. ${ }^{8}$ The 'direct activation' model proposes that certain BH3 proteins, such as tBid, Bim and evtl. Puma, directly bind and activate Bax and Bak irrespective of whether they are previously held in check by Bcl-2like survival factors or not. In this model the 'direct activator' BH3 proteins trigger membrane insertion and activation of Bax ('embedded together'), therefore shifting the equilibrium of cytosolic Bax toward the MOM. (b) Llambi et al. ${ }^{9}$ recently proposed a unified model by defining so-called 'MODES' that sequester pro-apoptotic Bcl-2 family members. In MODE 1, Bax and Bak are not kept in check by Bcl-2-like survival factors. Rather these survival factors sequester the 'direct activator' BH3 proteins tBid, Bim and evtl. Puma. Despite their high affinity, these interactions are reversible, allowing free 'direct activators' to persist but unable to activate Bax/Bak. Under high-stress conditions, however, they increase in amount and/or activity (due to equilibrium binding) until they directly bind and activate Bax/Bak without necessarily having been pre-bound to Bcl-2like survival factors ('direct activation' model as in panel a). Moreover, 'derepressor' BH3 proteins, such as Noxa, Bad, Bmf and so on, could displace activator BH3 proteins from Bcl-2-like survival factors, although this has not been experimentally proven yet (denoted by asterisks (*)). In MODE 2 active Bax and Bak are sequestered by Bcl-2-like proteins. This repression is more effective than MODE 1 allowing Bax/Bak activation only when BH3 proteins ('director activators' or 'derepressors') displace them from Bcl-2like survival factors. Westphal et al. ${ }^{4}$ also propose a MODE 0 by which Bcl-x $x_{L}$ regulates 'retrotranslocation' of Bax into the cytosol in healthy cells. The mechanism is unknown as no physical interaction between the two molecules has yet been demonstrated. It is also not known whether 'retrotranslocation' is mediated by membrane-bound or cytoplasmic Bcl- $\mathrm{x}_{\mathrm{L}}$. Irrespective of the model (a) or MODE (b) of regulation apoptosis proceeds when (1): Bax and Bak form a protein-lined (proteinaceous) pore consisting of several Bax/Bak molecules (multimers) or (2): monomeric Bax or Bak initially form a small lipidic pore that is later enlarged by Bax/Bak multimerization (as suggested by the use of nanodiscs in Volkmann et al. ${ }^{3}$ ). For further details see text 
interaction site at the hydrophobic groove. Bax/Bak would then undergo conformational changes and become activated in an autonomous manner (Figure 1a, left half). The problem with this model is that interactions between $\mathrm{Bcl}$-2-like survival factors and Bax are rarely seen in healthy cells ${ }^{8}$ because they localize to different subcellular compartments (Bax in the cytosol and Bcl-2-like survival factors in the MOM). Thus at least for Bax, the model does not sufficiently explain the activation mechanism (but see discussion on MODE 0 below and Figure 1b, right half). Even interactions of Bcl-2-like survival factors and MOM-residing Bak are not consistently detected in healthy cells. An alternative explanation of the 'derepression' model suggested that it is not Bax/Bak that are bound to Bcl-2-like survival factors in healthy cells but the 'direct activator' BH3 proteins tBid, Bim and Puma. Thus, these $\mathrm{BH} 3$ proteins would have to be consistently present in

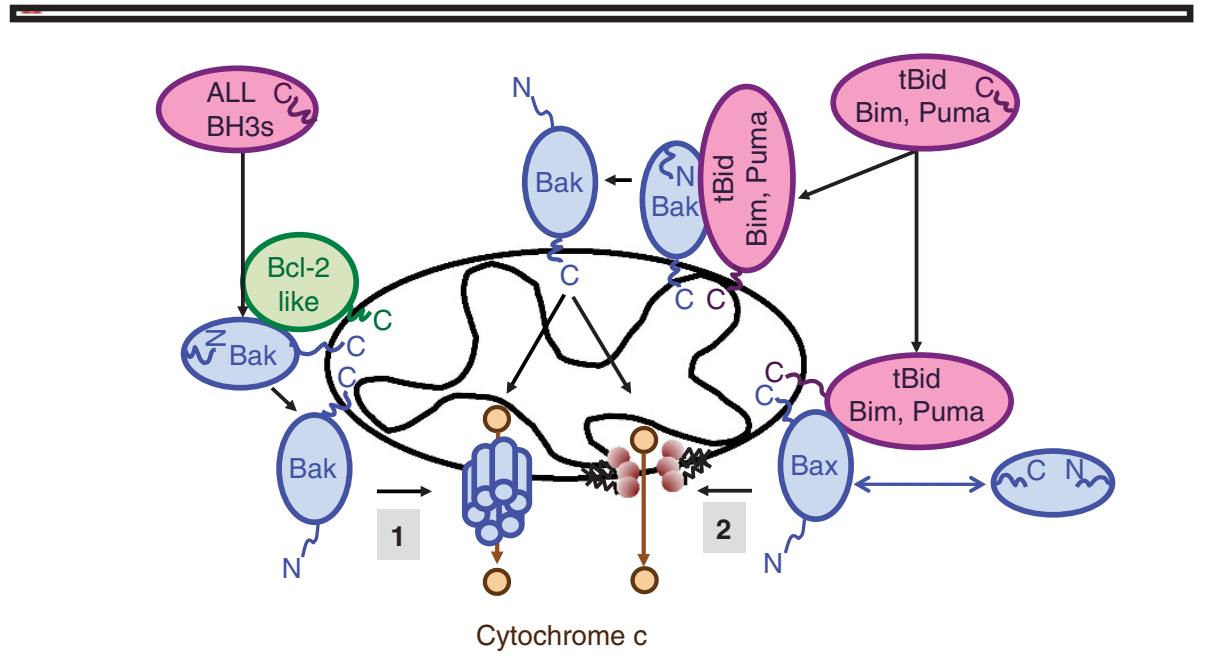

MODE 1:

Bcl-2-like survival factors sequester tBid, Bim and/or Puma.

When they increase in amount or are displaced by Noxa, Bad, Bmf, etc. they directly activate Bax/Bak
MODE 2:

Bcl-2-like survival factors sequester active Bax and Bak until displaced by BH3-proteins (all) (more effective than MODE 1).

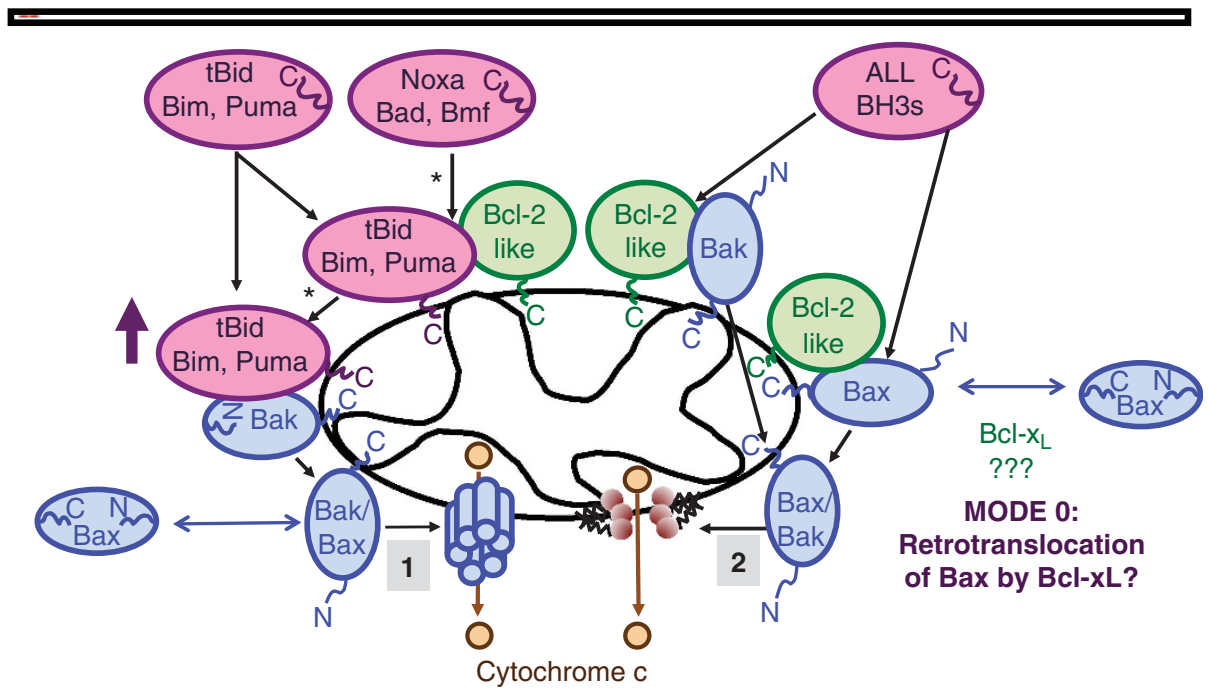


these cells. In most cases, Bim and Puma are, however, only transcriptionally induced upon apoptotic stimuli, and Bid needs to be cleaved by caspase- 8 into tBid in order to bind Bcl-2-like survival factors. Alternatively, tBid, Bim and Puma may quickly bind to Bcl-2-like survival factors after apoptotic induction. The 'derepression' action then means that other BH3 proteins (the 'derepressors'), such as Noxa, Bad, Bmf, Bik or Hrk, release the tBid, Bim and Puma activators from Bcl-2-like survival factors by competitive binding to the hydrophobic pocket. The attractive feature of such a model is that it provides a mechanism for cells to respond to mildto-moderate stress without dying. The problem with such a mechanism is that it has never been proven experimentally, and given the similar or even lower affinity of the 'derepressor' $\mathrm{BH} 3$ proteins for $\mathrm{Bcl}-2$-like survival factor binding, it is difficult to understand how they can displace the more tightly bound 'direct activators' Bim, Puma or tBid. This basically leaves us with the mechanisms that $\mathrm{tBid}$, Bim and, maybe also, Puma directly activate Bax and/or Bak after their transcriptional induction and perhaps further posttranslational modification ('direct activation' model). In this case, their interaction with Bcl-2-like survival factors may be more important as an inhibition mechanism. If they always saturate the prosurvival factors before there is significant binding to effector proteins, then further induction or activation of apoptosis would be required to produce more $\mathrm{BH} 3$ proteins to directly activate $\mathrm{Bax}$ and Bak (Figure 1b, left half). In the 'derepression' model, Bak, but not necessarily Bax, is bound to Bcl-2-like survival factors and can be released by 'any' BH3 protein that is activated in apoptotic cells (Figure 1a, left half). In this case the 'derepressor' BH3 proteins can only derepress Bak, but not directly activate Bak or Bax. For Bax, the 'derepression' mechanism still has to be defined. Perhaps, once Bax reaches the MOM and inserts into the membrane, it is prone to being activated by the 'direct activator' $\mathrm{BH} 3$ proteins or by other biochemical events.

Llambi et al. ${ }^{9}$ have recently proposed two different modes, MODE 1 and MODE 2, by which $\mathrm{BH} 3$ proteins and Bax/Bak are sequestered by $\mathrm{Bcl}-2$-like survival factors. Activation of Bax/Bak and apoptosis then ensues when MODE 1 and/or 2 are inhibited. Unfortunately, Westphal et al. ${ }^{4}$ use the terms MODE 1 and 2 in opposite ways; the sequestration processes as 'MODE 1 and 2 inhibitions' and the Bax/Bak activation steps as 'MODE 1 and 2 derepressions'. According to Llambi et al., ${ }^{9}$ in MODE 1, Bcl-2-like survival factors sequester 'direct activator' BH3 proteins (tBid, Bim and Puma; Figure 1b, left half). The authors propose that although the affinities of these interactions are high, they may be reversible, allowing some of the 'direct activators' to persist in a free form but at a level negligible to activate Bax and Bak. During high apoptotic stress, however, their concentrations or active forms would increase through transcriptional or posttranscriptional mechanisms so that they can directly activate Bax and Bak (Figure 1b, left half) as proposed in the 'direct activation' model (Figure 1a, right half). Whether 'derepressor' $\mathrm{BH} 3$ proteins (Noxa, Bad, Bmf, etc.) can displace the pre-bound 'direct activators' from Bcl-2-like survival factors at any time (Figure 1b, left half, asterisk) has not be proven by MODE 1 either, leaving this mechanism purely hypothetical. Westphal et al. ${ }^{4}$ take only this hypothetical displacement mechanism into account but dismiss the real sense of MODE 1 inhibition, which is direct Bax/Bak activation by excess tBid, Bim and Puma in apoptotic cells (Figure 1b, left half). In MODE 2, Bcl2-like survival factors sequester Bax/Bak and this repression seems to be more efficient that MODE 1 (sequestration of $\mathrm{BH} 3$ proteins). Upon binding of 'any' $\mathrm{BH} 3$ protein ('direct activator' and 'derepressor' types) to Bcl-2-like survival factors in apoptotic cells, Bax and Bak are released and activated autonomously or by the additional physical contact with a 'direct activator' $\mathrm{BH} 3$ protein (Figure 1b, right half).

Westphal et al. ${ }^{4}$ propose an additional mode, MODE 0 to explain the particular activation of Bax (Figure 1b, left half). This mode is based on two recent publications by the Youle ${ }^{10}$ and Gilmore ${ }^{11}$ groups that, in healthy cells Bax is in equilibrium between the cytosol and the MOM. Whenever a Bax molecule arrives on the MOM, it is 'retrotranslocated' to the cytosol and $\mathrm{Bcl}-2$-like survival factors such as Bcl- $\mathrm{x}_{\mathrm{L}}$ seem to have a crucial role in this reshuttling process (Figure 1b, left half). It has, however, remained unclear how $\mathrm{Bcl}-\mathrm{x}_{\mathrm{L}}$ performs such an action as no direct physical interaction with Bax in the cytosol or on the MOM has been biochemically detected so far in healthy cells. ${ }^{8}$ Billen et al. ${ }^{12}$ reported that a novel region of $\mathrm{Bcl}-\mathrm{x}_{\mathrm{L}}$ interferes with Bax binding to liposomes causing it to 'retrotranslocate', and thereby suggesting that a transient interaction between the proteins may shift the equilibrium for Bax binding to favor the cytoplasmic form. Alternatively, not yet identified molecules may participate in the regulation of the equilibrium of Bax between the cytosol and the MOM. The addition of MODE 0 to MODES 1 and 2 provides a more complete set of less ambiguous descriptors for the dominant interactions regulating apoptosis. Moreover, all three modes are consistent with both the recently proposed 'unified' and the 'embedded together' models for apoptosis regulation. The primary difference between these models is that the former explicitly states that MODE 2 is dominant, whereas the latter states that all of the interactions are governed by complex regulatable equilibria allowing any mode to be dominant depending on the physiological situation (both models are discussed in detail in Shamas-Din et al. ${ }^{13}$ ).

In summary, after years of research we still do not know how exactly Bax and Bak are activated in apoptotic cells. It is likely that in some respects all models may represent aspects of pathways that can act alone or in parallel. Alternatively, there might be a fundamental difference in how Bax and Bak are activated. In which case the two permeabilization processes may have to be modeled separately. The results of Bax and/or Bak activation are both obvious (permeabilization of the outer mitochondrial membrane) and obscure (the nature and regulation of the permeabilizing structure remaining controversial).

\section{What are the limitations of our experimental models?}

High-resolution structures have so far been obtained only for soluble forms of Bcl-2 family proteins. Data for complexes is even more limited with most including a relatively large fragment of only one protein bound to a peptide derived from another. The relatively low affinities reported for the various interactions virtually assure that in real membranes there are a variety of interconvertable complexes. Thus, it seems likely 
that inferences will have to be extracted across platforms for the foreseeable future. Differences between liposomes and planar bilayers will likely translate into kinetic and thermodynamic differences in the way the proteins interact but hopefully will not change the fundamental molecular mechanism(s). Moreover, it is likely that we have not yet identified all of the proteins involved. The recent identification of Mtch2 as a catalyst of tBid-mediated $\mathrm{MOMP}^{14}$ not only casts doubt on kinetic measurements made in liposomes lacking this protein, but may also explain the mysterious requirement for large amounts of cardiolipin in liposome-based MOMP assays. Alternatively, it could be that membrane curvature or lipid subdomains such as rafts may be equally important. Complicating matters further, Mtch2 is homologous to cardiolipin binding proteins suggesting that the effect of this protein on tBid may be indirect through reorganization of membrane lipids. Such a role could have profound effects on the assembly or physical properties of a lipidic pore in the membrane. Furthermore, cell-based assays are not a panacea. Most often such assays use transformed cells passaged in cultures that necessarily harbor defects in both apoptosis and anoikis. Finally, culture conditions including growth on plastic, exposure to serum, high oxygen and fluorescent lighting are all likely to provoke abnormal responses particularly to cell stress and intoxication that trigger apoptosis. However, the mechanistic details needed to exploit the $\mathrm{Bcl}-2$ family therapeutically cannot be revealed in animal studies. Integration of many techniques together with the development of quantitative predictive models will be essential to both understand and eventually manipulate MOMP.

\section{Conflict of Interest}

The authors declare no conflict of interest.

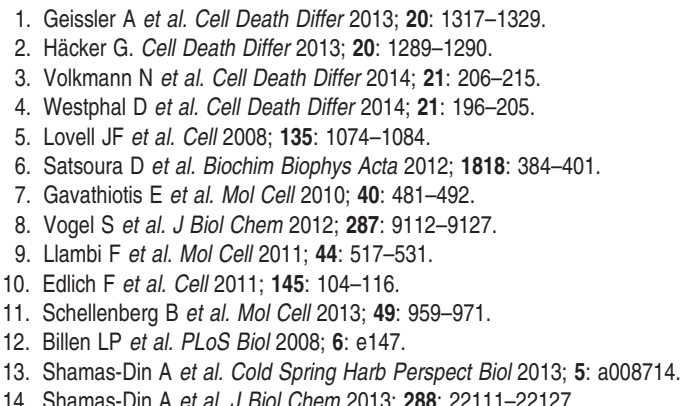

1. Geissler A et al. Cell Death Differ 2013; 20: 1317-1329.

7. Gavathiotis E et al. Mol Cell 2010; 40: 481-492.

8. Vogel S et al. J Biol Chem 2012; 287: 9112-9127.

11. Schellenberg B et al. Mol Cell 2013; 49: 959-971.

14. Shamas-Din A et al. J Biol Chem 2013; 288: 22111-22127. 